ance, analgesia, and perhaps local treatment with povidone-iodine. It is more difficult to predict the course in the over $50 \mathrm{~s}$, but in only 12 of the 55 did the pain last for more than a week or two. Whether idoxuridine in dimethyl sulphoxide produced better results than povidone-iodine seemed dubious, but it tends to be used in the elderly. Adequate analgesia is vital, but surely for the majority acyclovir does seem more than is necessary.

A O Chase

\section{Bristol BS5 8QY}

** We sent this letter to Wellcome's senior medical adviser, who replies below-ED, BMF.

SIR,-Intravenous acyclovir has been approved for the treatment of herpes zoster infections for some time. Zovirax tablets (acyclovir) have now been approved for the treatment of acute herpes zoster by the Committee on Safety of Medicines. We do not suggest that every case of shingles should be treated with acyclovir, but for those patients who in the doctor's opinion warrant treatment an effective and well tolerated oral treatment is now available.

E B Williams

The Wellcome Foundation Ltd,

Cheshire CW1 IUB

\section{Social adversity, low birth weight, and preterm delivery}

SIR, - The article by Dr Alan Stein and colleagues (1 August, p 291) raises an interesting point, but their analysis is of doubtful validity. Firstly, their test sample included only $3 \%$ of low birthweight infants and $3 \%$ of preterm infants; both these values are very low and significantly less than in the remainder of those born in the Radcliffe Infirmary and elsewhere. ${ }^{1}$ Secondly, their use of relative risk is curious; usually the proportion of the dependent variable is calculated in levels of the independen factors rather than vice versa. So, for example, for adverse life events they compare 3 out of $14(21 \%)$ with 71 out of $457(16 \%)$ and find no significance; we would have compared 3 out of $74(4 \%)$ with 11 out of $397(3 \%)$ and found a relative risk of $1 \cdot 4$, which, although not significant, may well be clinically important. In any case, with such a skewed small sample none of their analyses could ever actually refute any of the hypotheses considered.

Moreover, the observation by Dr Stein and coworkers that social class, adverse life events, and psychiatric state are not associated with birth outcome contrasts only with the studies that they quote and other simple breakdowns of raw birth weight by social class. As we have argued elsewhere, a more sophisticated and appropriate analysis has to control for sex, parity, and gestation, as well as maternal height, before testing for social class. $^{2}$

ROY A CARR-HILI

Centre for Health Economics,

University of York

Colin Pritchard

Social Paediatrics and Obstetrics Research Unit,

University of Glasgow

1 Office of Population Censuses and Surveys. Birthweight statistic 1985. London: OPCS, 1986. (OPCS Monitor, DH3, 86/2.)

2 Carr-Hill RA, Pritchard CW. The development and exploitation of empirical birhweight standards. London: Macmillan, 1985.

AUTHOR'S REPLY,-Unfortunately, Messrs CarrHill and Pritchard seem to have misunderstood much of our paper. They criticise an analysis that we did not even carry out and ignore at least half of our analyses when reaching their conclusions.

There is nothing "curious" about our analyses, and we did not even mention relative risks. The analyses to which they are presumably referring, the $\chi^{2}$ and Fisher's exact probability tests, are patently unaffected by whether the proportion of the dependent variable is examined in terms of the independent variable or vice versa. In fact, as Messrs Carr-Hill and Pritchard implicitly acknowledge, their own "less curious" method of analysis would lead to the same result in a significance test. Why their analysis leads them to believe that a non-significant difference may be of clinical importance when chance is as plausible an explanation we find baffling.

Of course, $3 \%$ is a low prevalence rate for low birth weight and preterm delivery in relation to other series, as we discuss fully in our paper. Furthermore, we state that "the small number of babies of low birth weight limited the interpretation" of the analyses using the dichotomous variables. We therefore carried out a second set of analyses, treating the two dependent variables as continuous - that is, considering the absolute birth weight and gestation of all 483 babies in the sample. Such a strategy increases the precision of the analyses, and we believe that it led us as far as possible to the possibility of refutation. How they can state that "none of their analyses could ever actually refute any of the hypothesis considered" is, again, difficult to understand.

Finally, as is well known in epidemiology, we could argue endlessly about which variables to include in an analysis. Whether the set of variables they prescribe is "more sophisticated and appropriate" than the ones we used is by no means self evident.

Department of Psychiatry,

University of Oxford

Oxford OX3 7JX

\section{Multiple births}

SIR,-Professor Eva Alberman (29 August, p 510) refers to a new survey launched by the Office of Population Censuses and Surveys of the long term physical, mental, and financial strains imposed on a family by a multiple birth. ${ }^{1}$ Though this initiative is welcome, it does draw attention to the neglected analysis of routinely collected statistics on multiple birth from a genetic epidemiology perspective.

Data on multiple births are available from 1938 onwards. ${ }^{2}$ The number of multiple births in England and Wales has varied between 5500 and 11000 a year, and with a stillbirth rate of only $18 /$ 1000 in $1985^{3}$ it may be assumed that a substantial series is available to explore the genetic and environmental contributions to the aetiology of particular diseases and possibly also to health related behaviour, such as alcohol consumption and smoking. The value of these data is, however, severely limited by our inability to distinguish between monozygous and dizygous births. Ethical and financial considerations make it unrealistic to suggest that blood specimens should be obtained for zygosity analysis, but a zygosity questionnaire that could show $80-90 \%$ agreement with red cell antigen testing might be sufficient for research purposes. ${ }^{4}$ My limited experience of studies of twins has taught me that families with twins are very willing to cooperate with research, ${ }^{5}$ although a system to protect individual families from excessive interference would need to be organised.

The range and quality of vital and health statistics produced by the Office of Population Censuses and Surveys is second to none in the world. One striking example, however, of a data set whose efficiency of collection has not been matched by imagination in analysis is that for multiple births, which has a potentially large contribution to make to our understanding of genetic epidemiology.

\section{R J RONA}

United Medical and Dental Schools of

Guy's and St Thomas's Hospitals,

London SE1 7EH

1 Office of Population Censuses and Surveys. Study of triplet and higher order births. London: Office of Population Censuses and Surveys, 1987.

Office of Population Censuses and Surveys. Birth statistics 1937-83. Historical series. London: HMSO, 1987. (Series FMI No 13.)

3 Office of Population Censuses and Surveys. Birth statistics 1985. London: HMSO, 1986. (Series FMI No 12.)

4 Lfung B, Fishbein S, Lindgren G. A comparison of growth in twins and singleton controls of matched age followed longitudinally from 10 to 18 years. Ann Hum Biol 1976;4:405-15.

5 Rona RJ, Angelico F, Antonini R, et al. Plasma cholesterol reponse to a change in dietary fat intake: a collaborative twin study. F Chronic Dis 1985;38:927-34.

\section{Skeletal dysplasias}

SIR,-Dr Ruth Wynne-Davies is incorrect in stating that achondroplasia cannot be diagnosed before 20-22 weeks' gestation (19 September, p 685). We have recently diagnosed two cases of achondroplasia at 18 weeks' gestation in low risk patients. In both cases femur shortening was found to be significantly below the 5th centile for gestation. ${ }^{1}$ Necropsy after elective termination of pregnancy, which included a full skeletal survey, confirmed the ultrasound diagnosis in each case. Both cases probably represented the homozygous or severe form of the disorder.

The heterozygous form of achondroplasia may also be diagnosed prenatally. Between 1978 and 1983,19 patients who were at risk of having a child with achondroplasia were referred to our unit for prenatal diagnosis. All of the four cases that were detected were diagnosed between 22 and 25 weeks' gestation, and no cases were missed. ${ }^{2}$ Each fetus had the heterozygous form of the disorder.

We would recommend that all high risk patients - those with a family history, personal history, or previous affected child-should have serial limb bone measurements taken, starting at 16 weeks' gestation. We would also encourage measurement of the fetal femur:foot length ratio when all fetuses at risk of dysplastic limb reduction are assessed. A femur:foot length ratio below the 5th centile can help to differentiate dysplastic skeletal growth from pseudolimb reduction due to constitutional smallness or intrauterine growth retardation. While some cases of achondroplasia may not be diagnosed until late in the second trimester, severe forms of this skeletal dysplasia may be detected before 20 weeks' gestation.

JAMes CAMPBell STUART CAMPBELL

Department of Obstetrics and Gynaecology,

King's College School of Medicine and Dentistry, London SE5 8RX

1 Queenan JT, O'Brien GD, Campbell S. Ultrasound measurement of fetal limb bones. Am $\mathcal{f}$ Obstet Gynecol 1980;136:297300.

2 Campbell S, Pearce JM. The prenatal diagnosis of fetal structural anomalies by ultrasound. In: Campbell $S$, ed. Ultrasound in obstetrics: recent advances, 10:3. London: W B Saunders, 1983:475-506.

\section{Cholesterolosis: a physical cause of} "functional" disorder

SIR,- We agree with the recommendations by Drs M R Jacyna and I A D Bouchier that patients with symptoms suggestive of biliary disease and no abnormality on cholecystography, ultrasono- 
graphy, or upper gastrointestinal endoscopy should undergo a cholecystokinin provocation test (12 September, p 619).

We have been using this test for over five years to identify patients in whom cholecystectomy will provide symptomatic relief. Our initial results were very encouraging. ${ }^{1}$ Our longer term results (mean follow up 34 months, range 12 to 68 months) in 59 patients who underwent cholecystectomy after a positive cholecystokinin test are equally encouraging; $64 \%$ of patients had complete symptomatic relief and a further $27 \%$ had pronounced symptomatic improvement, an overall satisfaction rate of $91 \%$. Only $9 \%$ of patients did not benefit from cholecystectomy.

Histopathological studies in our patients, however, did not always show abnormalities. Seventy per cent of patients had diseased gall bladders, most with the changes of chronic cholecystitis; less than $10 \%$ showed cholesterolosis; and $30 \%$ were microscopically normal. The presence of pathological changes within the gall bladder did not correlate with the clinical outcome. This may not be surprising as raised pressure in a normal gall bladder that cannot empty-for example, in the cystic duct syndrome-could conceivably cause pain.

We are anxious that cholecystokinin infusion may cause smooth muscle in the gut to contract, producing confusing pain. It has been our policy, however, to perform repeat cholecystokinin infusions after cholecystectomy, which does not cause pain. Concern has been expressed about the purity of some cholecystokinin preparations; we are currently evaluating synthetic cholecystokinin instead of the porcine hormone used in the series to date.

Recent research has shown that in patients with calculous biliary disease cholecystokinin receptors in the gall bladder wall are decreased. ${ }^{2}$ This down regulation of receptor sites correlates with decreased gall bladder motility and may be an early event in gall stone formation. We plan to examine these receptors in the patients in our series in an attempt to shed light on the pathogenesis of this syndrome.

M RHODES

R M R TAYLOR J R FARNDON

University of Newcastle upon Tyne,

Newcastle upon Tyne NE2 4HH

1 Lennard TWJ, Farndon JR, Taylor RMR. Acalculous biliary pain: diagnosis and selection for cholecsytectomy using the cholecystokinin test for pain reproduction. $\mathrm{Br} f$ Surg 1984;71:368-70.

2 Upp JR, Nealon WH, Singh P, et al. Correlation of cholecystokinin receptors with gallbladder contractility in patients with gallstones. Ann Surg 1987;6:641-8.

\section{Emergency phlebography service}

SIR,-As a practising radiologist, I must take issue with the view expressed by Dr I Sykes and colleagues (19 September, p 724) that arthrography is preferred to phlebography and, what is more, that satisfactory arthrography may be performed by resident medical staff without the support of a radiologist.

Firstly, establishing a diagnosis of deep vein thrombosis usually results in a change of management-namely, treatment with anticoagulantswhereas establishing a diagnosis of ruptured Baker's cyst does not alter the management. Indeed, arthrography will fail to show deep vein thrombosis associated with rupture of a Baker's cyst.

Secondly, both phlebography and arthrography are special procedures requiring specialist knowledge in their performance and interpretation. These investigations should be performed, whenever possible, by a skilled radiologist.

St Thomas's Hospital,

M F CREAGH

London SE1 7EH

\section{How to organise a clinical examination}

SIR,-Dr H N Cohen's article on organising a clinical examination (19 September, $p$ 714) is both apposite and amusing. I have arranged several conjoint examinations, MB finals, and MRCP examinations and know only too well the horrors that can ensue. I think, however, that some additional points need to be considered.

Firstly, the financial restrictions prevailing in and around London may mean that a spare ward is not available for examinations. This makes organisation more difficult but not impossible. The next best site is a large hall that can be adapted for use. It is extremely difficult, however, to gauge how many beds may be fitted into such an area. The eye deceives, and accurate measurements must be made to avoid embarrassment. As the hall may be some way from the main hospital complex particular care must be taken to give advanced notice of catering and linen needs. In addition, sudden illness must be anticipated, with resuscitation equipment at hand. Of course, special arrangements with porters may be necessary for transport of beds.

Secondly, I have found that it is often better to use a high percentage of outpatients who are known to be reliable. In view of the pressures on the National Health Service, it is unfair to use inpatients as this is bound to decrease bed turnover. If the examination is for MB finals many students will have seen a high percentage of suitable inpatients. If you choose outpatients it is important to make sure that they live locally otherwise you will have a large expenses bill.

Thirdly, the format of examinations differs from region to region, and the oral examination may be held on a different day to the clinical one. This means that the daily throughput of candidates and the demands on the invigilator are greater. The unhappy registrar who is running the examination can easily get lost, and it is most important to have a provisional plan that shows which candidate is allocated to which patient for a long case. As Dr Cohen mentions, patients do drop out at the last minute, and without a written record it is often impossible to remember where each candidate is and when he or she is due to meet the examiners.

D J B THOMAS

Mount Vernon Hospital,

\section{Passive smoking in utero: its effects on} neonatal appearance

SIR,-The photographs of babies' faces in the paper by Dr H F Stirling and colleagues (12 September, p 627) were a cheering sight on an otherwise bleak Saturday morning in Manchester. The observers in the experiment, however, were also shown of the whole of the babies' bodies, and there was a mean difference of $255 \mathrm{~g}$ between the weight of babies born to smoking mothers and those born to non-smokers. I challenge Dr Stirling and coworkers to repeat the experiment using only photographs of faces (supplied by me) and inform us whether selection by "gut feeling" still distinguishes babies of smoking mothers from hose of non-smoking mothers.

Dr Stirling and colleagues suggest that the result of this study might be helpful in health education. I would consider it not only deceitful but morally and ethically unacceptable for this study to be used for the health education of pregnant women while they are emotionally and physically nurturing a new life. Yet this is precisely the sort of research that enthusiastic and insensitive health educationalists (albeit a minority) would use to produce offensive posters stigmatising newborn babies' faces: "one look at her baby and I knew mum smoked."

The scientific basis of current health education propaganda in perinatal medicine is very weak. There is nothing of any substance to suggest that health education of the type that centres on the mothers' personal habits with respect to diet, smoking, and alcohol consumption has an important impact on the three dominant causes of perinatal mortality and morbidity in Britainvery preterm birth, perinatal asphyxia, and malformations.

By all means, let us have health education in perinatal medicine, but we must educate ourselves before we advise mothers. Until we have something to say about preventing the important causes of perinatal mortality and morbidity we must avoid letting our frustrations get the better of us and we must stop making pregnant women responsible for things we do not understand.

Malcolm L Chiswick

Saint Mary's Hospital,

SIR,-It might or might not be the case that passive smoking in utero affects the appearance of the neonate. The data collected by Dr H F Stirling and colleagues (12 September, p 627) do not, however, provide reliable evidence one way or the other; the statistical argument that they present is seriously flawed.

The crucial point is that photographs of the same 15 infants were shown to each of the 100 people interviewed, and so the resulting 1500 allocations cannot be analysed as independent. Dr Stirling and coworkers state that there was a difference in mean birth weight between the two groups $(3425 \mathrm{~g}$ for babies of non-smoking mothers, $3170 \mathrm{~g}$ for those of smokers; a difference of over $7 \%$ ). If those interviewed used this as a clue they would tend to make rather more correct guesses than incorrect ones, and this effect would accumulate over the 100 people. The fact that the difference in birth weights is not significant is irrelevant; the difference exists in the sample used. Similar arguments apply to body length and occipitofrontal circumference.

The data presented by $\mathrm{Dr}$ Stirling and colleagues may thus be explained quite adequately on the basis of what is already known about the effects of passive smoking in utero on the neonate. They also illustrate that the $B M \mathcal{F}$ has still not found a satisfactory method of screening its papers for statistical nonsense.

D O CHANTER S C COOK

Statistics Unit,

Huntingdon Research Centre,

Cambs PE18 6ES

AUTHORS' REPLY, -We are surprised by the strong opinions of our critics. We described an unexpected finding with no obvious explanation, a "difference" which needs to be confirmed or refuted by a further, more extended study. 